\title{
El autocuidado en mujeres posmenopáusicas con exceso en la nutrición
}

\author{
The Self-Care in postmenopausing women \\ with nutrition excess
}

MCE. Ignacio Grajales Alonso*, MCE. Erick Landeros Olvera**, MCE. Inés Tenahua Quitl***

y MCE. Gladis Linares Fleites****

*Alumno de la Maestría en Ciencias de Enfermería de la Benemérita Universidad Autónoma de Puebla (BUAP)

Profesor investigador de la Universidad de la Sierra Sur Oaxaca, ${ }^{*}$ Miembro del padrón de investigadores de la BUAP.

Estudiante de Doctorado en Ciencias de Enfermería en la UANL. Miembro del Capítulo Tau Alpha de Sigma Theta

Tau International, ${ }^{* *}$ Master en Ciencias de Enfermería. Profesora investigadora de la Universidad de la Sierra Sur

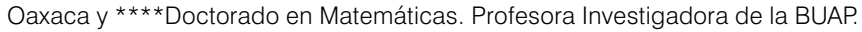

\section{Resumen}

México es considerado el segundo país con más obesidad en el mundo, desafortunadamente, las mujeres mueren cada vez más por Enfermedades Crónicas relacionadas al exceso de nutrición (obesidad) y otras desviaciones de la salud, por lo que este problema debe tratarse desde la prevención. Es aquí donde cobra importancia la utilización de conceptos teóricos como el modelo de Orem. Propósito: determinar la influencia de los factores condicionantes básicos sobre las capacidades y acciones de autocuidado y las relaciones teóricas entre estos dos conceptos, en mujeres de 45 a 60 años en etapa posmenopáusica con exceso en la nutrición. Metodología: el diseño fue de tipo correlacional. La muestra estuvo constituida por 142 mujeres de una comunidad rural. Resultados: los factores condicionantes básicos edad y la escolaridad influyen en las capacidades y acciones de autocuidado; esto refleja que la relación que existe entre la habilidad de ejercer el autocuidado, precede a la acción de realizarlas; las intervenciones de enfermería podrían estar dirigidas a fortalecer el empoderamiento de las habilidades para que se impulsen de forma automática la ejecución del cuidado. Discusión y conclusión: la relación entre capacidades y acciones de autocuidado es apoyada por los supuestos teóricos de la teoría del déficit de autocuidado y por validez estadística $(r=.869$, $p=.000$ ). Los resultados coinciden con otros estudios relacionados, sin embargo, estos se han desarrollado en diferentes poblaciones, con diferentes variables. Los resultados constituyen una fracción contributaria para el desarrollo de la práctica de enfermería basada en evidencia teórica.

Palabras clave: capacidades de autocuidado, acciones de autocuidado, factores condicionantes básicos, mujeres, exceso de nutrición.

\section{Abstract}

Mexico is considered the second most obese country in the world, and unfortunately more of our women are dying as a result of related nutritional chronic illnesses. Because of this, prevention becomes an important issue as it as been outlined in the Orem model. Purpose: to determine the influence of basic conditioning factors on self-care capacities and actions in women between 45 and 60 years old. Methodology: Correlational design with a sample of 142 rural community women. Results: consistent with self-care deficit theories, it was found that ability (and thus knowledge) mediates nutritional self-care actions (statistical validity $r=0.869 ; p=.000$ ). Discussion and conclusion: the findings resemble those of other similar theory-evidence based nursing studies; therefore we conclude that nursing interventions should strengthen women's self-care thrusting abilities.

Key words: self-care capacities, self-care actions, basic conditioning factors, women, nutrition excess. 


\section{INTRODUCCIÓN}

La Organización Mundial de la Salud informó que existen en el mundo más de 300 millones de personas que padecen obesidad (exceso de nutrición), además, advirtió que 17 millones mueren cada año por complicaciones a causa de este padecimiento(1,2). Esta epidemia estimó en personas adultas una prevalencia del $50 \%$ en el continente Americano(3). México es considerado el segundo país con más obesidad en el mundo, actualmente siete de cada diez mujeres adultas presentan un índice de masa corporal (IMC) mayor a 30 y se estima que para el año 2010 existan entre 8 y 14 millones de personas mayores de 35 años con esta desviación en la salud ${ }^{(4,5)}$. El problema radica en que el exceso de nutrición en conjunto con la condición hormonal, la edad, y la deficiencia de autocuidado como la falta ejercicio y el sedentarismo, representan factores de riesgo muy bien definidos que exacerban la aparición de otras desviaciones de la salud como la hipertensión arterial y la diabetes mellitus, ${ }^{(6,7)}$ primeras causas de muerte que constituyen un grave problema de salud pública con énfasis en las zonas indígenas o rurales, las cuales no cuentan con servicios especializados; la principal estrategia que debe retomarse es la prevención, principalmente en grupos potencialmente vulnerables como las mujeres entre los 45 y 60 años, porque después de esa edad, existe un mayor riesgo de padecer enfermedades crónicas. (8, 9, 10, 11).

Es urgente que la disciplina de enfermería teorice sobre las intervenciones más convenientes que favorezcan la salud y el bienestar, para que se inicie la práctica a través de la evidencia como una estrategia explícita y permanente para que en futuros programas puedan estructurar métodos específicos para prevenir enfermedades crónicas. Una de las formas de iniciar es utilizando modelos teóricos como el de Orem $^{(12)}$, específicamente la teoría del déficit de autocuidado, de la cual emana la agencia de autocuidado, dentro de esta, los conceptos teóricos que son susceptibles de medición son las Capacidades de Autocuidado y Acciones de Autocuidado. Teóricamente, estimar y comprender las Capacidades de Autocuidado para llevar incidir en el ejercicio de las Acciones de Autocuidado en las mujeres adultas con exceso de nutrición, podrían coadyuvar al sistema a reducir la morbilidad y la mortalidad por enfermedad crónica. Las Capacidades y las acciones de Autocuidado se adquieren de forma intencional y tienen un profundo significado cultural, debido a que están directamente influidas por el grupo social y las condiciones intrínsecas en las que está inmersa la mujer como son: la edad, la escolaridad, el nivel económico; elementos denominados Factores Condicionantes Básicos, los cuales pueden influir para incrementar o disminuir Capacidades y Acciones de Autocuidado. A pesar de que las Capacidades de Autocuidado pueden estar desarrolladas, pueden no operar, es decir, que por alguna razón las mujeres en la etapa de desarrollo entre los 45 y 60 años tienen habilidades para cuidar de su salud pero no hacen uso de ellas. Esta situación puede estar relacionada cuando las personas se perciben sanas, y no se preocupan por cuidarse, sino hasta que reconocen que su salud puede estar en peligro ya que no llevan a cabo Acciones de Autocuidado ${ }^{(13,14)}$. Algunas investigaciones han demostrando la relación de postulados teóricos a través de la obtención de estadísticos significativos de las Capacidades con las Acciones de Autocuidado $(15,16,17,18)$.

Referente a la relación teórica de los Factores Condicionantes Básicos con las Capacidades y Acciones de Autocuidado, diversas investigaciones han evidenciado que no todos tienen influencia que se explique estadísticamente. ${ }^{(15}$, $16,17,18,19,20,21,22)$

El estado del arte muestra estudios que relacionan las Capacidades de Autocuidado con los Factores Condicionantes Básicos, pero minoritariamente con investigaciones que específicamente hayan utilizado los conceptos de Capacidades y Acciones de Autocuidado en relación con los Factores Condicionantes Básicos. Adicionalmente las poblaciones de estudio abordan mayoritariamente ambos géneros, con enfermedad cardiovascular o metabólica declarada, y a pesar que las poblaciones son de nivel socioeconómico bajo, no pertenecen a áreas rurales. Bajo los resultados de ésta búsqueda surgió el siguiente propósito: determinar la influencia de los Factores Condicionantes Básicos seleccionados sobre las Capacidades y Acciones de Autocuidado y las relaciones teóricas entre estos dos conceptos, en mujeres con exceso de nutrición, posmenopáusicas y sin enfermedad cardiovascular agregada, residentes de una población rural.

Se abordaron las proposiciones teóricas que sirvieron como principios para contribuir a la verificación de los conceptos, para ello se plantearon las siguientes hipótesis:

- H1. Los Factores Condicionantes Básicos edad, escolaridad y nivel económico influyen en las Capacidades de Autocuidado en mujeres con exceso de nutrición.

- H2. Los Factores Condicionantes Básicos edad, escolaridad y nivel económico influyen en las Acciones de Autocuidado en mujeres con exceso de nutrición. 
- H3. Las Capacidades de Autocuidado se relacionan con las Acciones de Autocuidado en mujeres con exceso de nutrición.

\section{METODOLOGÍA}

El diseño del estudio fue descriptivo, correlacional de alcance transversal|(23). La muestra estuvo constituida por 142 mujeres en etapa posmenopáusica de 45 a 65 años de edad con obesidad de la comunidad rural de San Andrés Azumiatla del Estado de Puebla en México. Dicha comunidad se caracteriza por tener un alto grado de analfabetismo, actividad migratoria, escasez de servicios básicos sanitarios, hacinamiento en hogares, fauna nociva e ingresos económicos bajos. El muestreo se calculó por análisis de potencia con una p $05^{(24)}$. Para la medición de las variables se aplicó la cédula de Factores Condicionantes Básicos para personas con obesidad y un instrumento para estimar las Capacidades y Acciones de Autocuidado. La cédula de Factores Condicionantes Básicos consta de 19 preguntas para determinar datos generales; los factores que determina son: edad, escolaridad y nivel económico; antecedentes personales y familiares sobre factores de riesgo y registro de variables clínicas ${ }^{(25)}$. El instrumento "Uno mismo como encargado del cuidado"; consta de 40 reactivos, de éstos, 23 reactivos midieron las Acciones de Autocuidado y 17 las Capacidades Autocuidado. El patrón de respuesta incluyó seis opciones tipo Likert: 1 "Totalmente de acuerdo" y 6 "Totalmente en desacuerdo". Cada respuesta se sumó para producir un puntaje total; para fines de medición los resultados se invirtieron, así, el valor máximo es de 40 y el valor mínimo es de 240; mientras más bajo fue el puntaje total, más alto son los niveles de Capacidades y Acciones de Autocuidado percibidos ${ }^{(26)}$. La determinación de presión arterial, colesterolemia e IMC se realizaron bajo consideración de los criterios de la OMS, y las Normas Oficiales Mexicanas (NOM) $)^{(27,28,29)}$. Para la recolección de datos se recorrieron los sectores 4, 5 y 6 de la población por manzanas conforme a la técnica de manecillas del reloj para la identificación de hogares y de estos mujeres de 45 a 65 años. Esta investigación se llevó a cabo de acuerdo a las disposiciones de la Ley General de Salud Capítulo Quinto en materia de investigación para la salud(30); así mismo, se obtuvo la aprobación del Comité de Investigación y Ética de la Facultad de Enfermería de la Benemérita Universidad Autónoma de Puebla.

Para el análisis de los datos se utilizó el programa estadístico SPSS versión 12.0 ${ }^{(31)}$. Para las variables estudia- das se utilizó la estadística descriptiva e inferencial. En las hipótesis 1 y 2 se utilizó la regresión lineal múltiple y para la hipótesis 3 la correlación. Previamente se construyeron índices en una escala de 0 a 100 y se aplicó la prueba de normalidad (Kolmogorov-Smirnov) para determinar la factibilidad de utilizar estadísticos paramétricos en el análisis de la hipótesis 3. La confiabilidad de los instrumentos se obtuvo con el coeficiente de Alpha de Cronbach.

\section{RESULTADOS}

La muestra se compuso de 142 mujeres de 45 a 60 años. La edad promedio fue de 53.46 años (DE $=6.67$ ). La escolaridad promedio fue de 1.59 años (valor máximo de 12 y un mínimo de 1). El nivel socioeconómico un 57\% percibe menos de dos salarios mínimos. Con respecto a la medición del IMC mostró que las mujeres presentaron

\section{TABLA 1.}

Frecuencia y porcentaje de las variables clínicas del estado de salud.

\begin{tabular}{llll}
\hline Variable & Clasificación & $\mathbf{f}$ & $\%$ \\
\hline Obesidad & Grado I & 80 & 56 \\
& Grado II & 45 & 32 \\
& Grado III & 17 & 12 \\
\hline
\end{tabular}

Fuente: Cédula de factores condicionantes básicos para personas con obesidad (sección: Desviaciones de la salud). $n=142$

\section{TABLA 2.}

Clasificación del IMC en mujeres con exceso de nutrición.

\begin{tabular}{llcc}
\hline Variable & Clasificación & $\mathbf{f}$ & $\%$ \\
\hline Presión arterial & Óptima & 119 & 83.8 \\
& & & \\
& Normal & 7 & 4.9 \\
& & & \\
& Normal alta & 12 & 8.5 \\
Colesterol & $\begin{array}{l}\text { Normal } \\
\text { dl) }\end{array}$ & 142 & 100 \\
\hline
\end{tabular}

Fuente: Cédula de Factores Condicionantes Básicos para personas con obesidad (sección: Desviaciones de la salud). 
una $M d=30.45$, variable determinante para constatar en toda la muestra la presencia de obesidad (desviación de su la salud), variable de criterio de inclusión. Así mismo, para tener una muestra más homogénea, se precisó que toda la muestra estuviera en el estado de desarrollo posmenopáusico, sin enfermedad cardiovascular agregada que pudiera sesgar los resultados, por ello se determinó la presión arterial y la colesterolemia para incluir solo aquellas mujeres con límites normales. (Tabla 1 y 2).

La confiabilidad del instrumento se determinó por medio del coeficiente Alpha de Cronbach, se realizó por escalas, Capacidades de Autocuidado obtuvo .84 y Acciones de Autocuidado obtuvo .93

Para comprobar la hipótesis 1 y 2 , se utilizó el modelo de regresión lineal múltiple. El resultado encontrado fue significativo obteniendo una $F(3,138)=9.851, p=.000 \mathrm{R}^{2}$ ajustada .158 y una $F(3,138)=6.414, p=.000 \mathrm{R}^{2}$ ajustada .103 respectivamente por lo que ambas hipótesis se aceptaron. Los factores condicionantes básicos que tuvieron significancia estadística sobre las Capacidades y Acciones de Autocuidado fueron la edad y la escolaridad.

En la hipótesis 3, se utilizó la correlación de Pearson. El modelo fue significativo, se observó una correlación alta entre las Capacidades y Acciones de Autocuidado con una $r$ $=.869$ y una

$p=.000$, por lo que dicha hipótesis se aceptó. (Grafico 1)

\section{GRÁFICO 1}

Relación de las Capacidades con las Acciones de autocuidado en mujeres con exceso en la nutrición, posmenopáusicas y sin enfermedad cardiovascular agregada.

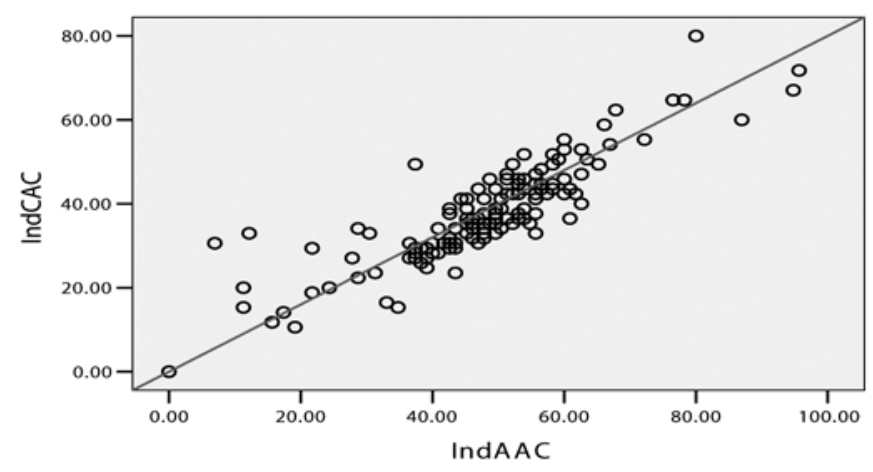

Fuente: "Uno Mismo como Encargado del Cuidado"

\section{DISCUSIÓN}

De acuerdo a la hipótesis uno, los resultados obtenidos sustentaron que la edad y escolaridad contribuyeron para determinar la influencia sobre las Capacidades de Autocuidado. Estas derivaciones coinciden con diversos autores, quienes encontraron que la edad influye en las Capacidades de Autocuidado $(14,15,16,19,20)$. Los hallazgos concuerdan con la base teórica de Orem, quien afirma que la edad es un aspecto de tipo cronológico y un proceso de maduración que influye en el desarrollo Capacidades de Autocuidado para otorgar a las personas las habilidades necesarias para cuidar de su salud. Sin embargo, considerando que el rango de edad de la muestra osciló entre 45 y 60 años, es posible que el proceso de maduración para favorecer el autocuidado esté más desarrollado a pesar de presentar un cambio en el estado de salud (obesidad); situando de esta manera a la edad como un factor que en esta muestra teóricamente es contradictorio al obtener una significancia estadística cuando el grupo de mujeres posee un IMC elevado ( $\mathrm{Md}=30.45$ ), en otras palabras, la etapa de maduración de esta muestra no influye en el desarrollo de las Capacidades de Autocuidado como teóricamente se indica, simplemente las capacidades no se desarrollan y no operan, porque no se percibe la necesidad de cuidar de la salud si no se tiene algún malestar físico o dolor y la obesidad no lo representa, situación parecida

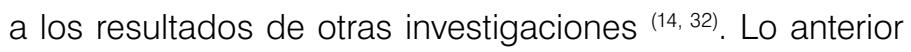
hace pensar que el exceso de nutrición no representa un riesgo para la salud en esta muestra y que no se ha adquirido conciencia de las posibles enfermedades crónicas que se pueden desarrollar a partir de tener obesidad, situación que se debe abordar desde el nivel preventivo, antes de que aparezcan enfermedades cardiometabólicas agregadas.

En lo que se refiere al nivel económico la influencia estadística no fue contundente sin embargo, en otras investigaciones el nivel económico fue significativo ${ }^{(18,19,20)}$. Dentro del modelo teórico, esta variable no se menciona específicamente como un Factor Condicionante Básico, pero tiene una relación con el factor de adecuación y disponibilidad de los recursos, el cual, teóricamente establece que la disponibilidad de recursos y su coste determina que los individuos favorezcan medidas de autocuidado. Una posible explicación de este hallazgo es que la economía deteriorada para esta población rural a lo largo de su vida ha sido una situación normal, por lo que no ha repercutido en su autocuidado; es decir, si nunca se han tenido los recursos, nunca se han utilizado, ¿cómo se puede hacer uso de servicios básicos sanitarios si son escasos y no se consideran?. 
De acuerdo a los resultados de la hipótesis dos, los Factores Condicionantes Básicos edad y escolaridad influyen en las Acciones de Autocuidado. Esta relevancia concuerda con otra investigación donde se encontró que ambos factores influyeron estadísticamente sobre las Acciones de Autocuidado ${ }^{(15)}$. Otros resultados son similares con respecto a la variable edad, no obstante la escolaridad ${ }^{(21)}$. Al respecto de esta última los resultados de esta investigación coinciden con la asociación estadísticamente significativa de los trabajos de otros investigadores ${ }^{(17,18)}$. Así mismo estos resultados se sustentaron con la afirmación teórica de Orem: a mayor edad las personas desarrollan una serie de disposiciones para ocuparse en formas de Acciones de Autocuidado intencionadas, es decir, aprenden qué hacer y qué no hacer en áreas progresivamente mayores de la vida humana. Puede suponerse que las habilidades de autocuidado en las mujeres de 45 a 60 años seleccionadas en este estudio, se encuentran completamente desarrolladas para poder ejecutar acciones para el cuidado de su salud. Con respecto a la escolaridad Orem señala que el aprendizaje es determinante para desarrollar habilidades y con ello la realización de acciones que garantizan de forma gradual el autocuidado. Empero, en esta muestra el nivel de escolaridad fue extremadamente bajo (característica particular de esta comunidad rural), y el estadístico demostró una asociación positiva, por lo que se puede interpretar que a menor escolaridad menor es la Acción de Autocuidado. No se asegura que las Acciones de Autocuidado se operacionalizen eficientemente, porque a pesar de tener una significancia estadística, el índice de Acciones de Autocuidado es relativamente bajo ( $\mathrm{Md}=48.12$ ). Los resultados de las hipótesis uno y dos son similares, tanto la escolaridad como la edad contribuyen con la asociación de los conceptos teóricos implicados. De la misma manera el nivel económico no influyó sobre las Acciones de Autocuidado, resultado que muestra diferencias con diversos autores ${ }^{(17,18,21,22)}$. Para concluir sobre la hipótesis tres que sustentó la relación entre las Capacidades y Acciones de Autocuidado en esta muestra, los resultados mostraron una congruencia teórica con los postulados de Orem: las personas entre mayor nivel de Capacidades de Autocuidado tienden a tener una mejor operacionalización de sus habilidades reflejándose en un nivel adecuado de Acciones de Autocuidado. Estos hallazgos son congruentes con algunos autores quienes encontraron que a mayor nivel de Capacidades de Autocuidado, el adulto ejecuta niveles más altos de Acciones de Autocuidado( ${ }^{(15,16,17,18)}$. Este resultado demuestra lo que teóri- camente Orem señala, para realizar una acción, se necesita poseer un impulso (Capacidad de Autocuidado), y cuando el ser humano las lleva a cabo de forma operacional, se establece la acción para su autocuidado. La verificación de los postulados teóricos específicamente en este trabajo han demostrado algunas similitudes con los resultados de estudios relacionados, sin embargo, las características de esta muestra difieren con las demás investigaciones, porque sus hallazgos se han obtenido de muestras heterogéneas, sus resultados se han generalizado con respecto al género y las características de los sujetos de estudio muestran enfermedades crónico degenerativas ya establecidas, lo que demuestra la importancia de esta investigación al considerarse bajo una relación epidemiológica y fisiológica la etapa de posmenopausia y obesidad como factores de riesgo para enfermedad crónica. La contribución principal de este trabajo es comprender cómo es que se establece las relaciones de las variables implicadas para que enfermería pueda incurrir bajo evidencia en la prevención de enfermedades

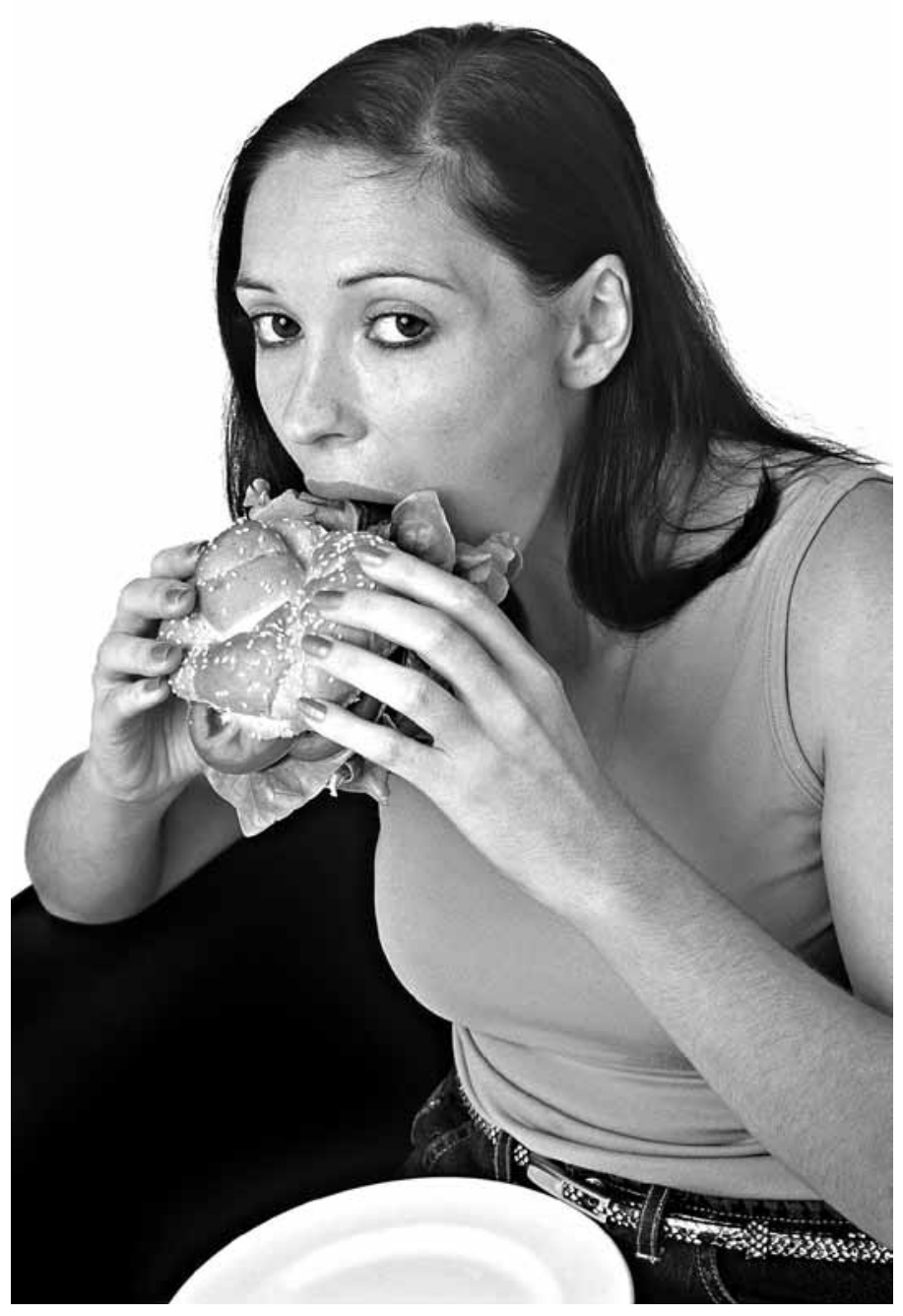


crónicas en este grupo específico. Por otra parte en América Latina los indicadores epidemiológicos confirman que la principal causa de muerte en mujeres de los 60 años son las enfermedades crónicas como la hipertensión arterial, la Diabetes Mellitus, ${ }^{(3,8,33)}$ lo que indica justamente que enfermería tiene que reforzar el autocuidado previo a la edad de riesgo (de los 45 a los 60 años -posmenopáusia-), con énfasis en las mujeres con obesidad para contribuir a la delimitación de los daños a la salud de las adultas mayores. El equipo interdisciplinario de salud tiene la obligación de ofrecer una atención especial para promover las acciones de salud antes de que se desarrolle la enfermedad crónica y evitar gastos catastróficos de salud al sistema y a las familias.

\section{CONCLUSIONES}

Específicamente los Factores Condicionantes Básicos que influyeron en la Capacidades y Acciones de Autocuidado fueron la edad y la escolaridad, no obstante el nivel económico a pesar de que la comunidad a la que pertenece la muestra carece de varios recursos, características particulares de las zonas rurales mexicanas.

Los hallazgos en este estudio mostraron que las Capacidades de Autocuidado se relacionan de forma significativa con las Acciones de Autocuidado en mujeres con exceso de nutrición, posmenopáusicas y sin enfermedad cardiovascular agregada.

\section{RECOMENDACIONES}

Realizar estudios en esta población con instrumentos validados que midan sus hábitos alimenticios a través de un diseño de alcance longitudinal.

Realizar estudios de intervención de manera interdisciplinaria para ofrecer una atención dirigida a la prevención de la obesidad en esta población para evitar la aparición de enfermedades crónicas y que contribuyan a mejorar la práctica del autocuidado.

Utilizar estudios mixtos o cualitativos relacionados al estilo de vida de las personas de esta comunidad para comprender de una forma complementaria el fenómeno de la obesidad.

\section{REFERENCIAS BIBLIOGRÁFICAS}

1. Organización Mundial de la Salud. Obesidad y sobrepeso 2006; Recuperado el 15 de noviembre de 2006, de http:// www.who.int/mediacentre/factsheets/fs311/es/indez/html.

2. Sánchez CP, Pichardo OE, López RP. Epidemiología de la obesidad. Gac. Med. México 2004; 140 (2): 3-20.
3. Organización Panamericana de la Salud. Salud en las Américas: Washington, D.C.: Publicación Científica y Técnica 2007; No. 622. Recuperado el 30 de octubre de 2007 de www.crid.or.cr/ digitalizacion/pdf/spa/doc16711/doc16711.htm - 7k.

4. Villa RA, Escobedo HM, Méndez SN. Estimación y proyección de la prevalencia de obesidad en México a través de la mortalidad por enfermedades asociadas. Gac. Med. México 2004; 140 (2): 21-25.

5. Olaiz G, Rivera J, Shamah T, Rojas R, Villalpando S, Hernández M, "et al". Encuesta Nacional de salud nutrición (ENSA). 2006

6. Brochu M, Poehlman T, Ades A. Obesity, body fat distribution, and coronary disease. J. Cardiopulm Rehabil. 2000; 20: 96108.

7. Ginsberg, N. Insulin resistance and cardiovascular disease. J. Clin Inv. 2000; 106 (4): 453-458.

8. Barquera S. Análisis crítico de la mala nutrición en el adulto. Salud Pública de México. Instituto Nacional de Salud Pública. Cuernvaca, México 2004; 49: 273-275.

9. Bastías AE, Sanhueza AO. Conductas de autocuidado y manifestaciones perimenopáusicas en mujeres de la comuna de concepción en Chile. Cienc. Enferm.2004; X (1): 41-56.

10. Lugones BM, Quintana RT, Cruz OY. Climaterio y menopausia: importancia de su atención en el nivel primario. Rev. Cubana Med. Gen. Integr. 2001; 13 (5): 494-503.

11. Stramba BM, Priori SG. Estrategias actuales para reducir el impacto de las enfermedades cardiovasculares en la mujer. Rev. Esp. Cardiol. 2006; 59 (11): 1.

12. Orem DE. Nursing: Concepts of practice. $5^{\mathrm{a}}$ ed. Ed. Mosby, St. Louis. 1995.

13. Salas SS, Alzaga BM, Landeros OE, Chávez DR. Testimonios de la mujer con angina. Index de Enferm. 2003; XII, (42): 9-13.

14. Landeros OE, Gallegos CE. Capacidades de autocuidado y percepción del estado de salud en personas con obesidad y sin obesidad. Rev. Mex. Enferm. Cardiol. 2005; 13 (1-2); 20-24.

15. Bañuelos CP, Gallegos CE. Autocuidado y control en adultos mayores con diabetes. Desarrollo Científ. Enferm. México 2001; 9 (4): 100-106.

16. Bernabé $\mathrm{CH}$. Capacidades de autocuidado en el paciente con diálisis peritoneal continua ambulatoria. México. En prensa 2004.

17. Tenahua QI, Landeros OE, Linares FG, Grajales, Al. Capacidades y Acciones de Autocuidado en personas con Factores de Riesgo Cardiovascular. Rev. Esp. Enferm. Cardiol. 2007; Año XIV (41): 19-24. 
18. Landeros OE, Tenahua QI, Salazar TJ, Jurabe T, Grajales AI. Obesidad y autocuidado en mujeres de una comunidad rural mexicana. Rev. Horiz Enferm. Chile 2007; 18 (2): 11-20.

19. Magaña CM. Influencia de los Factores Condicionantes Básicos en las Capacidades de Autocuidado en Pacientes con Hipertensión Arterial Sistémica. México. En prensa 2005.

20. Gallegos CE. Validez y Confiabilidad de la Versión en Español de la Escala: Valoración de las Capacidades de Autocuidado. Rev. Desarrollo Científ. Enferm. México 1998; 6 (9): 260-265.

21. Alamilla BM, García TG. Autocuidado y factores de riesgo de enfermedad cardiovascular en adultos de 25 a 60 años. Rev. Desarrollo Científ. Enferm. México 2007; 15 (7): 294298.

22. Alamilla BM, Canto BF, Chable HS, Moo MA. Factores condicionantes básicos sobre acciones de autocuidado en personas con diabetes mellitus tipo 2. Rev. Desarrollo Científ. Enferm. México 2004; 12(1): 9-13.

23. Polit, D.F, Beck ChT. Nursing Research $7^{a}$ ed. USA: Ed. Lippincott Williams \& Wilkins. 2004.

24. Polit D.F. Data Analysis \& Statistics for Nursing Research. USA: Ed. Prentice Hall. 1996.

25. Grajales Al, Landeros OE. Cédula de factores condicionantes básicos para personas con obesidad. Manuscrito no publicado. Facultad de Enfermería de la Benemérita Universidad Autónoma de Puebla 2007.

26. Geden E, Taylor S. Construct and empirical validity of the selfas- carer inventory. Nursing Research. 1991; 40 (1): 47-50.
27. Secretaría de Salud. Norma Oficial Mexicana NOM-174SSA1-1998. Para el manejo integral de la obesidad 2005.

28. Secretaría de Salud. Norma Oficial Mexicana NOM-030SSA2-1999. Prevención, tratamiento y control de la hipertensión arterial 2005.

29. Secretaría de Salud. Norma Oficial Mexicana NOM-037SSA2-2002. Para la prevención, tratamiento y control de las dislipidemias 2005.

30. Secretaría de Salud. Ley General de Salud Reglamento de la Ley de Salud en materia de Investigación para la Salud 2007. 35-37. Recuperado el 23 de abril de 2007 de www. salud.gob.mx/unidades/cdi/legis/lgs/index-indice.htm - 1k

31. Pallant J. SPSS Survival Manual. $2^{\text {a }}$ ed. Nueva, York, NY, EE. UU.: Ed. McGraw-Hill. 2006.

32. Soriano SM, Arrioja MG, Tlalpan HM. Percepción del estado de salud y su influencia en las capacidades de autocuidado en personas con síndrome coronario. Rev. Enferm. Cardiol. Mex. México 2005; 13, (1-2): 25-30.

33. Secretaría de Salud. Por un México sano: construyendo alianzas para una mejor salud. Programa Nacional de Salud 2005-2012. México 2005; 18-30. Recuperado el 30 de octubre de 2007. www. portal.salud.gob.mx/sites/salud/ descargas/pdf/pnscap1.pdf.

\section{DIRECCIÓN PARA CORRESPONDENCIA}

MCE. Erick Landeros Olvera: erick_landeros@mexico.com

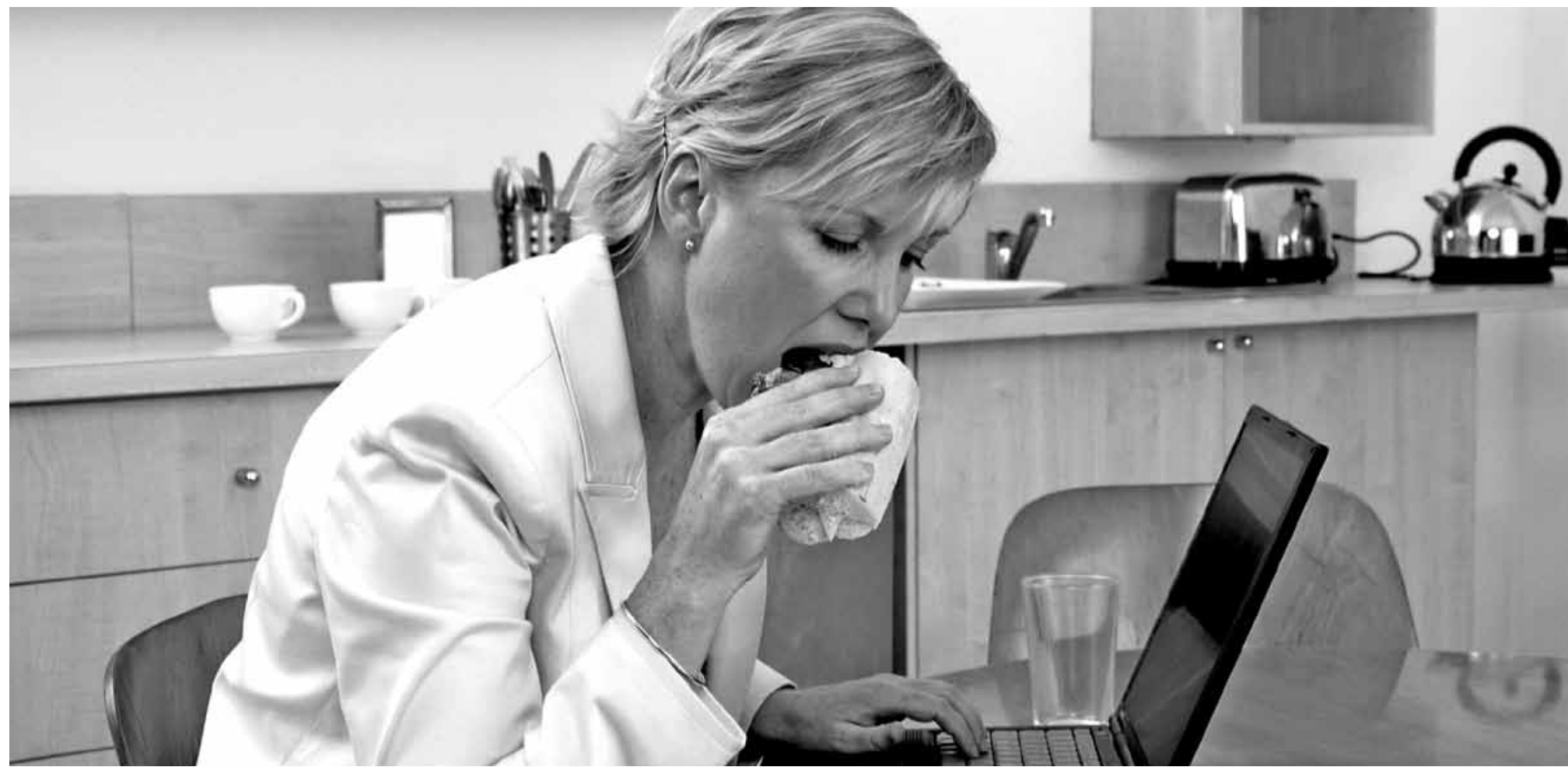

